\title{
CERTAIN SEMIGROUPS EMBEDDABLE IN TOPOLOGICAL GROUPS
}

\author{
HENERI A. M. DZINOTYIWEYI
}

(Received 18 December 1980)

Communicated by Gavin Brown

\begin{abstract}
In this paper we study commutative topological semigroups $S$ admitting an absolutely continuous measure. When $S$ is cancellative we show that $S$ admits a weaker topology $\mathscr{T}$ with respect to which $(S, \mathscr{T})$ is embeddable as a subsemigroup with non-empty interior in some locally compact topological group. As a consequence, we deduce certain results related to the existence of invariant measures on $S$ and for a large class of locally compact topological semigroups $S$, we associate $S$ with some useful topological subsemigroup of a locally compact group.
\end{abstract}

1980 Mathematics subject classification (Amer. Math. Soc.): 22 A 20, 43 A 05.

\section{Introduction}

Let $S$ be a commutative cancellative topological semigroup. The embedding of $S$ in a topological group $G$ such that $S$ has non-empty interior in $G$ has been studied in a number of publications-see for example Rothman (1960), Rigelhof (1971) and Paalman-de-Miranda (1964). In all these publications $S$ satisfies the property that $x U$ is an open subset whenever $U$ is an open subset of $S$ and $x \in S$; a property not necessarily satisfied by many useful subsemigroups with non-empty interior in a topological group.

When $S$ admits an invariant measure (that is a positive regular Borel measure $\mu$ such that $\mu(x C)=\mu(C)$ for all compact $C \subset S$ and $x \in S)$, Paterson (1977) used the invariant measure to define a topology on $S$ with respect to which $S$ (endowed with the new topology) is embeddable as a subsemigroup with non-empty interior

C) Copyright Australian Mathematical Society 1982 
in some locally compact group. Motivated by this result we do the same for $S$ only known to support an absolutely continuous measure and consequently show that such a semigroup $S$ admits an invariant measure. Further, for certain general commutative locally compact topological semigroups $S$ admitting an absolutely continuous measure we associate topological subsemigroups $S_{\rho}$ of a topological group in such a way that certain properties of $S$ carry over to $S_{\rho}$.

The plan of this paper is as follows: in Section 2 we clarify our notation and collect together some preliminary results. Our main results are presented in Section 3.

We ae indebted to Drs. J. W. Baker and A. L. T. Paterson for discussions which eventually led us to these studies.

\section{Preliminaries}

Unless otherwise stated, by a topological semigroup $S$ we mean a semigroup $S$ endowed with a Hausdorff topology with respect to which the semigroup operation $(x, y) \rightarrow x y$ is a jointly continuous mapping of $S \times S$ into $S$.

For any subsets $A, B,\{x\}$ of a semigroup $S$ we write $A B, A^{-1} B, x^{-1} B$ and $A^{-1} x$ in place of $\{a b: a \in A$ and $b \in B\},\{y \in S: a y \in B$ for some $a \in A\},\{x\}^{-1} B$ and $A^{-1}\{x\}$, respectively. We say $S$ is cancellative if $x y=x z$ or $y x=z x$ implies $y=z$ $(x, y, z \in S)$.

Now let $S$ be a topological semigroup for the remainder of this section. We denote the set of all bounded complex-valued Borel measures on $S$ by $M(S)$, the set of all bounded complex-valued continuous functions on $S$ by $C(S)$. For each $\nu \in M(S)$ we take $|\nu|$ to be the measure arising from the total variation of $\nu$, $\operatorname{supp}(\nu):=\{x \in S$ : if $V$ is any open neighbourhood of $x$ then $|\nu|(V)>0\}$ and for all $x \in S$ we let $\bar{x}$ denote the point mass at $x$.

Following Dzinotyiweyi (1978a) we say $S$ is a C-distinguished topological semigroup if the real-valued members of $C(S)$ separate points of $S$ and thus note that, for such an $S$, we have that $M(S)$ is a normed algebra under the usual total variation norm and with convolution multiplication given by

$$
\nu * \mu(f):=\int f(x y) d \nu(x) d \mu(y) \quad(\nu, \mu \in M(S) \text { and } f \in C(S)) .
$$

We define $M_{a}(S):=\{\nu \in M(S)\}:$ the maps $x \rightarrow|\nu|\left(x^{-1} C\right)$ and $x \rightarrow|\nu|\left(C x^{-1}\right)$ of $S$ into $\mathbf{R}$ are continuous for all compact $C \subset S\}$ and say $\nu \in M(S)$ is an absolutely continuous measure if $\nu \in M_{a}(S)$. The closure of $U\{\operatorname{supp}(\nu): \nu \in$ $\left.M_{a}(S)\right\}$ is called the foundation of $M_{a}(S)$ and we denote it by $F_{a}(S)$. 
2.1. Theorem (see Dzinotyiweyi (1978a), Theorem 4.8). If $S$ is a C-distinguished topological semigroup, then $M_{a}(S)$ is a norm closed subalgebra of $M(S)$ such that

(i) $\nu * \mu, \mu * \nu \in M_{a}(S)$ for all $\nu \in M_{a}(S)$ and $\mu \in M(S)$;

(ii) $\nu \ll|\mu|$ and $\mu \in M_{a}(S)$ imply $\nu \in M_{a}(S)$ for all $\nu \in M(S)$.

For various results on $M_{a}(S)$ for the case where $S$ is locally compact we refer the interested reader to Baker and Baker (1970 and 1972), Dzinotyiweyi (1978b), Dzinotyiweyi and Sleijpen (1979), and Sleijpen (1976 and 1978).

Also the set $M_{e}(S):=\{\nu \in M(S)$ : given compact $C \subset S$ and $\varepsilon>0$, there is an open neighbourhood $W$ of $C$ such that $|\nu|\left(x^{-1}(W \backslash C)\right)<\varepsilon$ and $|\nu|\left((W \backslash C) x^{-1}\right)<\varepsilon$ for all $x$ in $\left.S\right\}$ is shown to be very closely related to $M_{a}(S)$ in Dzinotyiweyi (1978b), and Dzinotyiweyi and Sleijpen (1979). In particular we have

2.2. Proposition (see Dzinotyiweyi (1978b) for the case where $S$ is locally compact). If $S$ is any topological semigroup, then $M_{e}(S) \subseteq M_{a}(S)$.

Proof. Let $\nu \in M_{a}(S), C$ any compact subset of $S$ and $\left(x_{\alpha}\right)$ a net converging to $x$ in $S$. By Dzinotyiweyi (1978a), Lemma 2.1 (ii), the mapping $s \rightarrow|\nu|\left(s^{-1} C\right)$ of $S$ into $\mathbf{R}$ is continuous if

$$
\frac{\lim }{\alpha}|\nu|\left(x_{\alpha}^{-1} C\right) \geqslant|\nu|\left(x^{-1} C\right) .
$$

Now let $\varepsilon>0$ be given and choose an open neighbourhood $W$ of $C$ such that $|\nu|\left(y^{-1}(W \backslash C)\right)<\varepsilon$ for all $y$ in $S$. We then have

$$
\begin{aligned}
\frac{\lim }{\propto} & |\nu|\left(x_{\propto}^{-1} C\right)=\frac{\lim }{\propto}\left(|\nu|\left(x_{\propto}^{-1} W\right)-|\nu|\left(x_{\propto}^{-1}(W \backslash C)\right)\right) \\
& \geqslant \frac{\lim }{\propto}|\nu|\left(x_{\propto}^{-1} W\right)-\varepsilon \\
& \geqslant|\nu|\left(x^{-1} W\right)-\varepsilon \quad \text { by Dzinotyiweyi (1978a) Lemma 2.1 (i) } \\
& \geqslant|\nu|\left(x^{-1} C\right)-\varepsilon .
\end{aligned}
$$

Similarly the map $s \rightarrow|\nu|\left(C s^{-1}\right)$ of $S$ into $\mathbf{R}$ is continuous. Hence $M_{e}(S) \subseteq M_{a}(S)$.

\section{The main results}

Throughout this section $S$ stands for a commutative topological semigroup. Following Dzinotyiweyi (1978b), page 498, we define $\mathscr{T}$ to be the topology on the semigroup $S$ generated by the pseudometrics $\left\{d_{\nu, C}: \nu \in M_{a}(S)\right.$ and $C$ a compact 
subset of $S\}$ where

$$
d_{\nu, C}(x, y):=|\nu|\left(x^{-1} C \Delta y^{-1} C\right)
$$

We take $(S, \sigma)$ to be the semigroup $S$ endowed with the topology $\mathscr{T}$. We now have

3.1. THEOREM. Let the topological semigroup $S$ be cancellative and such that $M_{a}(S)$ is non-zero. Then

(i) $S$ is a $C$-distinguished topological semigroup.

(ii) $(S, \mathcal{G})$ is a topological semigroup with $\mathcal{G}$ weaker than the original topology of $S$.

(iii) $(S, \sigma)$ is embeddable as a topological subsemigroup with non-empty interior in some locally compact topological group $\left(G, \sigma^{\prime}\right)$.

(iv) There is a set $W \subset S$ such that $W$ is dense in $F_{a}(S)^{2}$ and $S y$ is a locally compact topological subsemigroup of $\left(G, \sigma^{\prime}\right)$ contained in the interior of $(S, \sigma)$ in $\left(G, \widetilde{\sigma}^{\prime}\right)$, for all $y \in W$. Further on such semigroups $S y$ the original topology and $\sigma^{\prime}$ coincide.

(v) Sv admits an invariant measure $\mu$ such that

$$
M_{e}(S v)=M_{a}(S v)=L(S v, \mu), \text { for all } v \in W .
$$

(vi) $M_{e}(S)=M_{a}(S)$.

Proof. (i) Suppose $x, y$ are distinct points of $S$ and choose a non-zero measure $\eta$ in $M_{a}(S)$. Let $v \in \operatorname{supp}(\eta)$ and note that $x v \neq y v$. There is an open neighbourhood $V$ of $v$ such that $x V \cap y V=\varnothing$. Now since $v \in \operatorname{supp}(\eta)$ it is trivial to deduce from Dzinotyiweyi (1978a), Lemma 2.2 and the Radon-Nikodym Theorem, that we can find a positive measure $\nu \in M_{a}(S)$ with $C:=\operatorname{supp}(\nu) \subset V$ and $C$ compact. Then $x C \cap y C=\varnothing$ and the function $f: s \rightarrow \nu\left(s^{-1}(x C)\right)$ is real-valued and continuous on $S$. Further

$$
\begin{aligned}
& f(x)=\nu\left(x^{-1}(x C)\right)=\nu(C)>0 \text { and } \\
& f(y)=\nu\left(y^{-1}(x C)\right)=\nu\left(y^{-1}(x C) \cap C\right)=0 .
\end{aligned}
$$

Hence $S$ is $C$-distinguished.

(ii) Since $S$ is $C$-distinguished, recalling Theorem 2.1 , one can easily produce an argument similar to that given in Dzinotyiweyi (1978b), Theorem 4.1, to show that multiplication in $(S, \widetilde{J})$ is separately continuous and $\widetilde{T}$ is weaker than the original topology on $S$. The rest of the proof is contained in the proof of item (iii) below.

(iii) Let $R$ be the equivalence relation on $S \times S$ given by

$$
(x, y) R(a, b) \text { if and only if } x b=y a \text {. }
$$


Now $G:=S \times S / R$ can (as well known) easily be shown to be an abelian group. For each $x \in S$ let $\tilde{x}:=\{(x y, y): y \in S\}$ and note that the map $\theta: S \rightarrow G$ given by $\theta(x):=\tilde{x}$ is a one-to-one homomorphism. As well known we may now identify $S$ with $\theta(S)$ and write elements of $G$ in the form $x^{-1} y$ tor some $x, y \in S$, where $x^{-1}$ denotes the inverse of $x$ in $G$.

Corresponding to each compact set $C \subset S$ and $\nu \in M_{a}(S)$ we define the mapping $d_{\nu, C}^{G}: G \times G \rightarrow \mathbf{R}$ by

$$
d_{\nu, C}^{G}\left(a^{-1} b, x^{-1} y\right):=|\nu|\left(b^{-1}(a C) \Delta y^{-1}(x C)\right)
$$

for all $a, b, x, y$ in $S$, where $b^{-1}(a C) \Delta y^{-1}(x C)$ is evaluated in $S$. It is trivial to verify that $d_{\nu, C}^{C}$ is a pseudo-metric such that if $g:=a^{-1} b$ and $h:=x^{-1} y$ are elements of $G$ contained in $S$ then

$$
d_{\nu, C}^{G}(g, h)=d_{\nu, C}(g, h) .
$$

Hence taking $\mathcal{T}^{\prime}$ to be the topology on $G$ generated by the pseudo-metrics $\left\{d_{v . C}^{G}\right.$ : $\nu \in M_{a}(S)$ and $C$ is a compact subset of $\left.S\right\}$ we have that $\mathscr{T}$ is the restriction of the topology $\mathcal{T}^{\prime}$ ' on $S$.

We now show that multiplication in $\left(G, \mathcal{T}^{\prime}\right)$ is separately continuous. Suppose $g_{\alpha} \rightarrow g$ in $\left(G, \mathscr{J}^{\prime}\right)$ and $h \in G$. We can write $g_{\alpha}:=a_{\alpha}^{-1} b_{\alpha}, g:=a^{-1} b$ and $h:=x^{-1} y$ for some elements $a_{\alpha}, b_{\alpha}, a, b, x, y$ in $S$. Then for any $\nu \in M_{a}(S)$ and compact $C \subset S$, we have that

$$
\begin{aligned}
& d_{\nu, C}^{G}\left(g_{\propto} h, g h\right)=d_{\nu, C}^{G}\left(\left(a_{\propto} x\right)^{-1}\left(b_{\propto} y\right),(a x)^{-1}(b y)\right), \\
& r=|\nu|\left(\left(b_{\propto} y\right)^{-1}\left(a_{\propto} x C\right) \Delta(b y)^{-1}(a x C)\right) \\
&=\bar{y} *|\nu|\left(b_{\propto}^{-1}\left(a_{\propto} x C\right) \Delta b^{-1}(a x C)\right) \\
&=d_{\bar{y} *|\nu|, x C}^{G}\left(g_{\propto}, g\right) \\
& \rightarrow 0 \quad \text { since } \bar{y} *|\nu| \in M_{a}(S) .
\end{aligned}
$$

Next we show that $\left(G, \mathscr{T}^{\prime}\right)$ is Hausdorff. Let $a^{-1} b$ and $x^{-1} y$ be any two distinct elements of $G$ (where, as before $a, b, x, y$ are elements of $S$ ). Then $a y \neq x b$. Let $\nu \in M_{a}(S)$ and $k \in S$ with $k \in \operatorname{supp}(\nu)$. Since $a y b k \neq x b b k$ we can choose a neighbourhood $U$ of $k$ such that $a y b U$ and $x b b U$ are disjoint. We can choose a compact set $K \subset U$ with $|\nu|(K)>0$. Then operating in $S$ we have (by the cancellative and commutative properties of $S$ )

$$
\begin{aligned}
y^{-1}(x b K) \cap a K & =y^{-1} b^{-1}(b x b K) \cap(b y)^{-1}(b y a K) \\
& \subseteq(b y)^{-1}(x b b U \cap a b y a U)=\varnothing .
\end{aligned}
$$


Thus taking $C:=b K$, we have $b^{-1}(a C) \backslash y^{-1}(x C)=a K$. Hence

$$
\begin{aligned}
d_{\bar{a} *|v|, C}^{G}\left(a^{-1} b, x^{-1} y\right) & =\bar{a} *|\nu|\left(b^{-1}(a C) \Delta y^{-1}(x C)\right) \\
& \geqslant \bar{a} *|\nu|(a K)=|\nu|(K)>0 .
\end{aligned}
$$

It follows that if $2 \varepsilon:=d_{\bar{a} *|v|, C}^{G}\left(a^{-1} b, x^{-1} y\right)$, then $\left\{g \in G: d_{\bar{a} *|v|, C}^{G}\left(a^{-1} b, g\right)<\varepsilon\right\}$ and $\left\{g \in G: d_{\bar{a}}^{G} \cdot|v|, C\left(x^{-1} y, g\right)<\varepsilon\right\}$ are two disjoint neighbourhoods of $a^{-1} b$ and $x^{-1} y$, respectively.

We now show that $\left(G, \mathcal{J}^{\prime}\right)$ contains a compact neighbourhood $F$ such that $F \subset(S, \Im)$ ). (In what follows unless otherwise stated, all sets of the form $A^{-1} B$ for $A, B \subset S$ are evaluated in $S$ and not $G$.) Take positive measures $\nu_{1}, \nu_{2}$ and compact sets $C_{1}, C_{2}$ as in the first paragraph of the proof of Dzinotyiweyi (1978a), Theorem 4.5. Note that $K:=C_{1} \cap y_{0} C_{2}^{-1}$ is a compact subset of $S$ with $\nu_{1}(K)>0$ and write $y:=a^{-1} b$ for some $a, b \in S$. Then taking $\varepsilon:=\nu_{1}(K)$ and noting that $b^{-1}\left(a y_{0} K\right)=K$, we have that

$$
\begin{aligned}
\left\{x^{-1} y \in G: d_{\nu_{1}, y_{0} K}^{G}\right. & \left.\left(x^{-1} y, y_{0}\right)<\varepsilon\right\} \\
& =\left\{x^{-1} y \in G: \nu_{1}\left(y^{-1}\left(x y_{0} K\right) \Delta b^{-1}\left(a y_{0} K\right)\right)<\varepsilon\right\} \\
& =\left\{x^{-1} y \in G: \nu_{1}\left(y^{-1}\left(x y_{0} K\right) \Delta K\right)<\varepsilon\right\} \\
& \subseteq\left\{x^{-1} y \in G: y^{-1}\left(x y_{0} K\right) \cap K \neq \varnothing\right\} \\
& \subseteq\left\{x^{-1} y \in G: y_{0} K \cap x^{-1}(y K) \neq \varnothing\right\} \\
& \subseteq y_{0} K K^{-1} \quad \text { with evaluation done in } G \\
& \subseteq y_{0} C_{1}\left(y_{0} C_{2}^{-1}\right)^{-1} \quad \text { with evaluation done in } G \\
& \subseteq C_{1} C_{2} .
\end{aligned}
$$

Hence $F:=C_{1} C_{2}$ is a compact neighbourhood of $y_{0}$ in $\left(G, \mathscr{T}^{\prime}\right)$ with $F \subset(S, \mathscr{T})$. (The preceding argument is related to that given in Paterson (1977), page 327.)

So $\left(G, \mathcal{T}^{\prime}\right)$ is locally compact and so by Ellis (1957), Theorem 2, we have that $\left(G, \mathcal{J}^{\prime}\right)$ is a locally compact topological group. That $(S, \mathcal{T})$ is a topological subsemigroup with non-empty interior in $\left(G, \mathcal{J}^{\prime}\right)$ is now clear.

(iv) Assume the notation of Dzinotyiweyi (1978a), page 331 and let $W$ be the set of all $y_{0}$ 's constructed there and note that $W$ is dense in $F_{a}(S)^{2}$. Now (as observed in the proof of (iii)) $F:=C_{1} C_{2}$ is a compact subset of $S$ which is a compact neighbourhood of $y_{0}$ in $\left(G, \widetilde{T}^{\prime}\right)$. For any $s$ in $S$ we thus have $s F$ a compact neighbourhood of $s y_{0}$ in $\left(G, \mathcal{J}^{\prime}\right)$ and hence in $S$ since $s F$ is a compact subset of $S$. Further $\mathcal{T}$ and the original topology of $S$ clearly coincide on $s F$. So item (iv) follows.

(v) Since $S v$ is a locally compact subset of $\left(G, \widetilde{J}^{\prime}\right)$ it is a Borel subset of $\left(G, \widetilde{J}^{\prime}\right)$, by Willard (1970), item 18.4. So taking $\mu$ to be the restriction of Haar measure on 
$S v$ we have an invariant measure on $S v$. For every Borel $B \subset S v$ and $x \in S v$ we clearly have

$$
\mu\left(x^{-1} B\right) \leqslant \mu(B) .
$$

Hence the Radon-Nikodym theorem and our definition of $M_{e}(S)$ gives $L(S v, \mu)$ $\subseteq M_{e}(S v)$. Our Proposition 2.2 says that $M_{e}(S v) \subseteq M_{a}(S v)$. So it remains to show that $M_{a}(S v) \subseteq L(S v, \mu)$. Let $\nu \in M_{a}(S v), d \in \operatorname{supp}(\nu)$ and $D$ any compact neighbourhood of $d$. Suppose for some compact $K \subset S v$ we have $\mu(K)=0$. It is easy to note that $|\nu| * \mu_{\mid D} \ll \mu$ and so

$$
\int|\nu|\left(K d x^{-1}\right) d|\nu|(x)=|\nu| * \mu_{\mid D}(K d)=0 .
$$

So $|\nu|\left(K d x^{-1}\right)=0(x \in \operatorname{supp}(\nu))$ since $x \rightarrow|\nu|\left(K d x^{-1}\right)$ is a non-nonnegative continuous function on $S v$. In particular we have

$$
|\nu|(K)=|\nu|\left(K d d^{-1}\right)=0
$$

and we are done.

(vi) By Proposition 2.2, we only need to show that $M_{a}(S) \subseteq M_{e}(S)$. Fix $\nu \in M_{a}(S)$ and $v \in W$, where $W$ is as in (v). Then $|\nu| * \bar{v} \in M_{e}(S v)$. Let $C$ be a compact subset of $S$ and $\varepsilon>0$. There is an open set $U$ containing $C v^{2}$ such that $U$ is open in $S$ (by (iv)) and

$$
|\nu| * \bar{v}\left(\left(U \backslash C v^{2}\right)(s v)^{-1}\right)<\varepsilon \quad(s \in S) .
$$

Now

$$
|\nu|\left(\left(U v^{-2} \backslash C\right) s^{-1}\right)=|\nu| * \bar{v}\left(\left(U \backslash C v^{2}\right)(s v)^{-1}\right) \quad(s \in S)
$$

(by cancellation) and so $\nu \in M_{e}(S)$ since $U v^{-2}$ is an open neighbourhood of $C$.

3.2. NoTES. When $S$ is a locally compact topological semigroup with an identity element and such that $S$ coincides with $F_{a}(S)$, Sleijpen (1976), Chapter X, proved (in a different way to our results) that there is a dense open subsemigroup $S_{1}$ of $S$ such that $S_{1}$ is topologically isomorphic to some open subsemigroup of a locally compact group.

Following Paterson (1977) $S$ is said to be an invariant measure semigroup if there exists a non-zero inner regular Borel measure $\mu$ on the $\sigma$-ring generated by the compact subsets of $S$ such that $\mu_{*}(U):=\sup \{\mu(C)$ : compact $C \subset U\}<\infty$ for $U$ in a neighbourhood base for $S$. It now follows from our Theorem 3.1 (iv) and Paterson (1977), Theorem 4 (i), that a cancellative $S$ with $M_{a}(S)$ non-zero is an invariant measure semigroup. 
3.3. We recall that $S$ is assumed to be commutative throughout this section. We define a relation $\rho$ on $S$ by

$$
x \rho y \quad \text { if and only if } x s=y s \text { for some } s \in S .
$$

One can easily note that $\rho$ is an equivalence relation such that if $x \rho y$ then $x z \rho y z$ for all $x, y, z \in S$. We denote the equivalence class of $x$ by $[x]$ and note that $[x]=\cup\left\{\{x s\} s^{-1}: s \in S\right\}$. We then have

$$
[x]=\{x\} \text { if and only if } S \text { is cancellative. }
$$

Let $S_{\rho}:=S / \rho=\{[x]: x \in S\}$. Clearly $S_{\rho}$ with multiplication given by $[x][y]=$ [xy] for all $x, y$ in $S$, is a cancellative commutative semigroup. We also denote the projection map of $S$ onto $S_{\rho}$ by $\rho$.

3.4. LeMMA. Let $S$ be a topological semigroup and $S_{\rho}$ be endowed with the quotient topology. Then if $W$ is a neighbourhood of $[w]$ in $S_{\rho}$, there is an open neighbourhood $O$ of $w$ in $S$ such that $[O]:=\{\rho(x): x \in O\}$ is a neighbourhood of $\rho(w)$ with $[O] \subseteq W$.

Proof. By definition of quotient topology $\rho^{-1}(W)$ is a neighbourhood of $w$. Let $O_{1}$ be any neighbourhood of $w$ with $O_{1} \subset \rho^{-1}(W)$. Then $O:=\cup\left\{\left(O_{1} s\right) s^{-1}\right.$ : $s \in S\} \subseteq \rho^{-1}(W)$ and $\rho^{-1}([O])=\rho^{-1}(\rho(O))=O$. Hence $O$ is the required neighbourhood.

3.5. TheOREM. Let $S$ be a topological semigroup and assume that $S_{\rho}$ is endowed with the quotient topology. Then

(i) $S_{\rho}$ is a commutative cancellative semigroup with jointly continuous multiplication;

(ii) if $S_{\rho}$ is Hausdorff and $S$ is locally compact and such that for all compact $C \subseteq S$ the set $\cup\left\{(C s) s^{-1}: s \in S\right\}$ is compact, then $S_{\rho}$ is a locally compact topological semigroup.

Proof. (i) In view of the remarks made in item 3.3 we only need to show that multiplication in $S_{\rho}$ is jointly continuous. To this end let $W$ be an open neighbourhood of $[x y]$ in $S_{p}$. Using the notation of Lemma 3.4 we can choose an open set $O_{1} \subset S$ with

$$
x y \in O_{1} \subset O \text { and }[O] \subset W .
$$

Now let $X_{1}, Y_{1}$ be neighbourhoods of $x, y$ (respectively) in $S$ such that $X_{1} Y_{1} \subset O_{1}$. Then defining $X, Y$ and $[X],[Y]$ with respect to $X_{1}, Y_{1}$ as done for $O$ and $[O]$ with respect to $O_{1}$ we note that

$$
X Y \subset \bigcup\left\{\{z s\} s^{-1}: z \in X_{1} Y_{1} \text { and } s \in S\right\} \subset O
$$


and hence $[X],[Y]$ are neighbourhoods of $[x],[y]$ such that $[X][Y] \subset[O] \subset W$. This completes our proof for item (i).

(ii) In view of item (i) and Kelley (1955), Theorems 3.12 and 5.20, we only need to show that the map $\rho: S \rightarrow S_{\rho}$ is closed. To this end let $F$ be a closed subset of $S$ and $\left(x_{\propto}\right)$ a net converging to $x$ in $S$ with $\left(x_{\alpha}\right) \subset \rho^{-1}(\rho(F))$. Then there are nets $\left(s_{\propto}\right) \subset S$ and $\left(f_{\propto}\right) \subset F$ such that

$$
x_{\propto} s_{\propto}=f_{\propto} s_{\propto} .
$$

Let $X$ be a compact neighbourhood of $x$ and hence note that eventually

$$
\left(f_{\propto}\right) \subseteq \bigcup_{\propto}\left\{\left(X s_{\propto}\right) s_{\alpha}^{-1}\right\} \subseteq T:=\bigcup\left\{(X s) s^{-1}: s \in S\right\} .
$$

Since $T$ is compact (and $F$ is closed) we may assume that $f_{\propto} \rightarrow f$ for some $f \in F$. By continuity of the quotient map we have $\rho\left(f_{\propto}\right) \rightarrow \rho(f)$ and $\rho\left(f_{\propto}\right)=\rho\left(x_{\propto}\right) \rightarrow$ $\rho(x)$. Since $S_{\rho}$ is Hausdorff it follows that $\rho(x)=\rho(f) \in \rho(F)$ and so $x \in$ $\rho^{-1}(\rho(F))$. Thus $\rho$ is closed.

3.6. Remarks. (i) One can easily note that if there is a continuous map $\tau$ of $S$ onto a cancellative topological semigroup $T$ then we can find a continuous map $\alpha$ of $S_{\rho}$ onto $T$ such that the following diagram commutes:

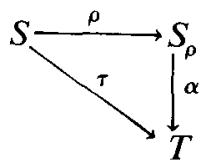

(ii) A case in which Theorem 3.5 is interesting is the following: Suppose the conditions stated in Theorem 3.5 (ii) are met and $M_{a}(S)$ is non-zero. Then a result of Sleijpen (1976), Theorem 3.10 says that $M_{a}\left(S_{\rho}\right)$ is non-zero. We thus obtain our Theorem 3.1 for the semigroup $S_{\rho}$.

(iii) Note that the "size" of our semigroup $S_{\rho}$ (when the conditions of Theorem 3.5 are met) is generally not close to $S$. For example take $S:=\{(x, y): x \in \mathbf{N}$ and $y \in[0, \infty)\}$ with the usual topology and multiplication given by

$$
(a, b)(x, y):=(a+x, \max \{b, y\}) \text {. }
$$

Now one can easily note that $S_{\rho}$ is isomorphic to the additive discrete semigroup N.

\section{References}

A. C. Baker and J. W. Baker (1970), 'Algebras of measures on a locally compact semigroup II', $J$. London Math. Soc. 2, 651-659.

A. C. Baker and J. W. Baker (1972), 'Algebras of measures on a locally compact semigroup III', $J$. London Math. Soc. 4, 685-695. 
H. A. M. Dzinotyiweyi (1978a), 'Algebras of measures on C-distinguished topological semigroups', Math. Proc. Cambridge Philos. Soc. 84, 323-336.

H. A. M. Dzinotyiweyi (1978b), 'On the analogue of the group algebra for locally compact semigroups', J. London Math. Soc. 17, 489-506.

H. A. M. Dzinotyiweyi and G. L. G. Sleijpen (1979), 'A note on measures on foundation semigroups with weakly compact orbits', Pacific J. Math. 81, 61-69.

R. Ellis (1957), 'Locally compact transformation groups', Duke Math. J. 24, 119-125.

J. L. Kelley (1955), General topology (Van Nostrand, Princeton, N.J.).

A. B. Paalman-de-Miranda (1970), Topological semigroups (Mathematical centre tracts, Amsterdam).

A. L. T. Paterson (1977), 'Invariant measure semigroups', Proc. London Math. Soc. 35, 313-332.

R. Rigelhoff (1971), 'Invariant measures on locally compact semigroups', Proc. Amer. Math. Soc. 28, $173-176$.

N. J. Rothman (1960), 'Embedding of topological semigroups', Math. Ann. 139. 197-203.

G. L. G. Sleijpen (1976), Convolution measure algebras on semigroups (Thesis, Katholieke Universiteit, Toernooiveld, Nijmegen).

G. L. G. Sleijpen (1978), 'Locally compact semigroups and continuous translation of measures', Proc. London Math. Soc. 37, 75-97.

S. Willard (1970), General topology (Addison-Wesley, Reading, Mass.).

Department of Mathematics

University of Zimbabwe

P. O. Box MP 167

Mount Pleasant, Salisbury

Zimbabwe 